\title{
METRIZABILITY OF LOCALLY COMPACT VECTOR SPACES
}

\author{
SETH WARNER
}

\begin{abstract}
By use of the theory of characters and the Pontryagin-van Kampen theorem, it is shown that if $E$ is a locally compact vector space over a discrete division ring $K$ of characteristic zero and if $\operatorname{dim}_{K} E<2^{\mathfrak{m}}$, where $\mathfrak{m}$ is the cardinality of $K$, then $E$ is metrizable.
\end{abstract}

The problem of determining whether a locally compact vector space over a discrete division ring is metrizable arises in the study of finite-dimensional locally compact vector spaces, because we have a fairly concrete picture of those that are metrizable: If $E$ is a finitedimensional, metrizable, indiscrete locally compact vector space over a discrete field $K$ and if $o$ is the smallest open subspace of $E$, then the topological additive group $D$ admits the structure of finite-dimensional topological vector space over the locally compact field $F$, where $F$ is either the real field $R$, the field $\boldsymbol{Q}_{q}$ of $q$-adic numbers, or the field $\boldsymbol{Z}_{p}((X))$ of power series over the field $\boldsymbol{Z}_{p}$ of integers modulo $p$, under a scalar multiplication satisfying $\alpha .(\lambda x)=\lambda(\alpha . x)$ for all $x \in E, \lambda \in K$, $\alpha \in F$; moreover, if $E$ is a topological algebra, then $\mathrm{o}$ is an ideal and $\alpha .(x y)=(\alpha . x) y, \alpha .(y x)=y(\alpha . x)$ for all $\alpha \in F, x \in 0, y \in A$; finally, $K$ is algebraically isomorphic to a subfield of finite codegree of a finite extension of $F$ [4, Theorems 3 and 5]. Here we shall consider the special case of this problem where the scalar field has characteristic zero.

First, we need a lower bound on the dimension of nonzero compact vector spaces. Let $K$ be a division ring, equipped with the discrete topology. We denote by $K^{\wedge}$ the (compact) character group of the discrete additive group $K$, made into a right topological vector space over $K$ by defining $u . \lambda: x \mapsto u(\lambda x)$ for all $u \in K^{\wedge}, \lambda \in K, x \in K[3$, Theorem 1].

THEOREM 1. If $K$ is an infinite division ring of cardinality $\mathfrak{m}$, then $\operatorname{dim}_{K} K^{\wedge}=2^{\mathfrak{m}}$.

Proof. Case 1. The characteristic of $K$ is zero. Then for some cardinal number $\mathfrak{n}$ the additive group of $K$ is isomorphic to $\mathbb{Q}^{(\mathfrak{n})}$, the

Received by the editors January 20, 1970.

AMS 1968 subject classifications. Primary 4601.

Key words and phrases. Topological vector space, topological algebra, locally compact, metrizability. 
direct sum of $\mathfrak{n}$ copies of the additive group $\boldsymbol{Q}$ of rationals, where $\mathfrak{n}=\mathfrak{m}$ if $\mathfrak{m}>\boldsymbol{\aleph}_{0}$ and where $1 \leqq \mathfrak{n} \leqq \boldsymbol{\aleph}_{0}$ if $\mathfrak{m}=\boldsymbol{\aleph}_{0}$. Hence $K^{\wedge}$ is topologically isomorphic to $\left(Q^{\wedge}\right)^{\mathfrak{n}}$, the cartesian product of $\mathfrak{n}$ copies of $\mathbf{Q}^{\wedge}[\mathbf{2}$, $(23.21)$, p. 364], and card $\left(Q^{\wedge}\right)=\mathfrak{c}\left[2,(25.4)\right.$, p. 404]. If $\mathfrak{m}>\boldsymbol{N}_{0}$, then $\operatorname{card}\left(K^{\wedge}\right)=c^{\mathfrak{n}}=\mathfrak{c}^{\mathfrak{m}}=2^{\mathfrak{m}}>\mathfrak{m}$, so $\operatorname{dim}_{K} K^{\wedge}=2^{\mathfrak{m}}$. If $\mathfrak{m}=\aleph_{0}$, then $\operatorname{card}\left(K^{\wedge}\right)$ $=\mathfrak{c}^{\mathfrak{n}}=\mathfrak{c}>\mathfrak{m}$, whence again $\operatorname{dim}_{K} K^{\wedge}=2^{\mathfrak{m}}$.

Case 2. The characteristic of $K$ is a prime $p$. Then the additive group of $K$ is isomorphic to $Z_{p}^{(\mathfrak{m})}$, so $K^{\wedge}$ is topologically isomorphic to $\left(\boldsymbol{Z}_{p}\right)^{\mathfrak{m}}$. Hence $\operatorname{card}\left(K^{\wedge}\right)=p^{\mathfrak{m}}=2^{\mathfrak{m}}>\mathfrak{m}$, so $\operatorname{dim}_{K} K^{\wedge}=2^{\mathfrak{m}}$.

As a consequence of Theorem 1 , we note that if $K$ is uncountable, then $K^{\wedge}$ is a nonmetrizable compact $K$-vector space of dimension $2^{\mathfrak{m}}$ [3, Theorem 8].

THEOREM 2. Let $K$ be a discrete division ring of characteristic zero. If $E$ is a locally compact, totally disconnected $K$-vector space, then $E$ is metrizable.

Proof. Let $Q$ be the prime field of $K$. By [2, (7.7), p. 62], $E$ contains a compact open subgroup $V$. Let $F=\bigcap\left\{\alpha V: \alpha \in Q^{*}\right\}$. Then $F$ is a compact vector space over $Q$ and hence is connected [3, Theorem 9]. Thus $F=(0)$. Hence, as $V$ is compact, for any neighborhood $W$ of zero there exist $\alpha_{1}, \ldots, \alpha_{n} \in Q^{*}$ such that $W \supseteq \alpha_{1} V \cap \ldots \cap \alpha_{n} V$. Therefore $\left\{\alpha_{1} V \cap \ldots \cap \alpha_{n} V: \alpha_{1}, \ldots, \alpha_{n} \in Q^{*}\right\}$ is a fundamental system of neighborhoods of zero in $E$; in particular, $E$ is metrizable.

THEOREM 3. Let $K$ be a discrete division ring of characteristic zero, and let $\mathfrak{m}=\operatorname{card}(K)$. If $E$ is a locally compact $K$-vector space and if $\operatorname{dim}_{K} E<2^{\mathfrak{m}}$, then $E$ is metrizable.

Proof. Let $C$ be the connected component of zero. By Theorem 2, $E / C$ is metrizable. By $[2,(\mathrm{e})$, p. 47], it therefore suffices to show that $C$ is metrizable. Hence we may assume that $E$ is connected. By the theorem of Pontryagin and van Kampen [2, (9.14), p. 95], the topological additive group $E$ is the topological direct sum of $R^{m}$ and $H$, where $H$ is a compact subgroup. Let $u$ be the (continuous) projection of $E$ on $R^{m}$ along $H$. If $h \in H$, then the closed additive subgroup $(\boldsymbol{Z} h)^{-}$generated by $h$ is compact as it is contained in $H$; if $\lambda \in K$, then $(\boldsymbol{Z} \lambda h)^{-}=\boldsymbol{\lambda}(\boldsymbol{Z} h)^{-}$, a compact subgroup, whence $u\left((\boldsymbol{Z} \boldsymbol{\lambda} h)^{-}\right)=(0)$ as $R^{m}$ contains no nonzero compact additive subgroups, and therefore $\lambda h \in(Z \lambda h)^{-} \subseteq H$. Hence $H$ is a vector subspace of $E$. By Theorem 1, [3, Theorem 6], and our hypothesis, $H=(0)$. Hence $E=R^{m}$ and thus is metrizable.

If $K$ is countable, we may improve Theorem 3:

Theorem 4. Assume the Continuum Hypothesis. If $K$ is a countable, discrete division ring of characteristic zero and if $E$ is a locally compact 
$K$-vector space such that $\operatorname{dim}_{K} E \leqq c$, then $E$ is metrizable.

Proof. As in the proof of Theorem 3, we may assume that $E$ is the topological direct sum of $\boldsymbol{R}^{m}$ and a compact subspace $H$. By [3, Theorem 6], $H$ is topologically isomorphic to the compact $K$-vector space $\left(K^{\wedge}\right)^{\mathfrak{n}}$, the cartesian product of $\mathfrak{n}$ copies of $K^{\wedge}$, for some cardinal number $\mathfrak{n}$. If $\mathfrak{n}>\boldsymbol{N}_{0}$, then $\operatorname{card}\left(K^{\wedge}\right)^{\mathfrak{n}}=\mathfrak{c}^{\mathfrak{n}}>\mathfrak{c}$, so $\operatorname{dim}_{K} E \geqq \operatorname{dim}_{K} H>\mathfrak{c}$, a contradiction. Hence $\mathfrak{n} \leqq \boldsymbol{N}_{0}$, so $H$ is metrizable as $K^{\wedge}$ is [3, Theorem $8]$. Thus $E$ is metrizable.

It is an open question whether similar theorems hold for locally compact vector spaces over fields of prime characteristic. At any rate, we may take care of the one-dimensional case:

THEOREM 5. If $E$ is an indiscrete, one-dimensional locally compact vector space over a discrete field $K$, then there is a topology on $K$ making $K$ into an indiscrete locally compact field and $E$ a topological vector space over $K$, so topologized; in particular, $E$ is metrizable.

Proof. The proof is similar to that of [3, Theorem 10]. We topologize $K$ so that $f: \lambda \mapsto \lambda a$ is a homeomorphism, where $a$ is a nonzero vector. Then $K$ is locally compact; $\lambda, \mu) \mapsto \lambda+\mu$ is continuous, since each of the maps $(\lambda, \mu) \mapsto(\lambda a, \mu a) \mapsto \lambda a+\mu a=(\lambda+\mu) a \mapsto \lambda+\mu$ is; and for each $\alpha \in K, \lambda \mapsto \alpha \lambda$ is continuous, since each of the maps $\lambda \mapsto \lambda a$ $\mapsto \alpha \lambda a \mapsto \alpha \lambda$ is. With the induced topology, the multiplicative group $K^{*}$ satisfies the hypotheses of Ellis's theorem [1, Theorem 2], so $K^{*}$ is a locally compact group. In particular, the mapping $(\lambda, \mu) \mapsto \lambda \mu$ is continuous at $(1,1)$; it is therefore also continuous at $(0,0)$, for if $V$ is a neighborhood of zero, there exists a neighborhood $U$ of zero such that $(1+U)(1+U) \subseteq 1+V$, whence $U U \subseteq U+U+U U=(1+U)(1+U)$ $-1 \subseteq V$. Therefore $K$ is an indiscrete locally compact field, so its topology is given by an absolute value; consequently, $E$ is also metrizable. Clearly $E$ is a topological vector space over $K$, as each of the maps $(\lambda, \mu a) \mapsto(\lambda, \mu) \mapsto \lambda \mu \mapsto \lambda \mu a$ is continuous.

\section{REFERENCES}

1. Robert Ellis, Locally compact transformation groups, Duke Math. J. 24 (1957), 119-125. MR 19, 561.

2. Edwin Hewitt and Kenneth A. Ross, Abstract harmonic analysis, Vol. I: Structure of topological groups. Integration theory, group representations, Die Grundlehren der math. Wissenschaften, Band 115, Academic Press, New York; SpringerVerlag, Berlin, 1963. MR 28 \#158.

3. Seth Warner, Compact and finite-dimensional locally compact vector spaces, Illinois J. Math. 13 (1969), 383-393. MR 39 \#3282.

4. - Locally compact commutative artinian rings, Illinois J. Math. (to appear).

Duke University, Durham, North Carolina 27706 